\title{
Conversion to Modified Duodenal Switch for Relieving Intractable Dumping Syndrome and Constipation After Laparoscopic Roux-en-Y Gastric Bypass
}

\author{
Chih-Kun Huang • Ming-Yu Wang • Ming-Che Hsin • \\ Po-Chih Chang
}

Published online: 21 February 2015

(C) Springer Science+Business Media New York 2015

\begin{abstract}
Background Dumping syndrome is not infrequent after laparoscopic Roux-en-Y gastric bypass and could result in dreaded complications, such as hypoglycemia or syncope. Those patients who failed medical therapy and diet modification, regarded as intractable dumping syndrome, may be considered as candidates for revisional surgery. Herein, we make a video presentation of laparoscopic revisional procedure for intractable dumping syndrome with unsatisfactory weight loss.

Methods A 32-year-old, morbidly obese woman (initial body mass index, $53.3 \mathrm{~kg} / \mathrm{m}^{2}$ ) presented with dumping syndrome 17 months after initial laparoscopic Roux-en-Y gastric bypass. She underwent nutritional counseling, strict diet modification, and medication treatment but failed. In addition, the patient complained of worsening constipation and insufficient weight loss (body mass index, $36 \mathrm{~kg} / \mathrm{m}^{2}$ ). Laparoscopic revisional procedure with modified duodenal switch was conducted to relieve her intractable condition.

Results The procedure took $260 \mathrm{~min}$ without any intraoperative complication. Blood loss was $100 \mathrm{~mL}$ The patient had an uneventful postoperative course and the postoperative hospital stay was 5 days. The uncomfortable symptoms relieved successfully after the revisional surgery.
\end{abstract}

Electronic supplementary material The online version of this article (doi:10.1007/s11695-015-1607-9) contains supplementary material, which is available to authorized users.

C.-K. Huang $\cdot$ M.-C. Hsin $\cdot$ P.-C. Chang $(\bowtie)$

Bariatric and Metabolic International Surgery Center, No. 1, Yi-Da

Road, Jiao-Su Village, Yan-Chao Distinct, Kaohsiung City 824,

Taiwan

e-mail: dr.changpochih@hotmail.com

C.-K. Huang $\cdot$ M.-Y. Wang $\cdot$ M.-C. Hsin $\cdot$ P.-C. Chang

Division of General Surgery, Department of Surgery, E-Da hospital/

I-Shou University, Kaohsiung City, Taiwan
Conclusions Though long-term follow-up is warranted to draw a definite conclusion, modified duodenal switch with pyloric restoration and shortening bowel length remains an acceptable revisional procedure to relive intractable dumping syndrome and constipation in our patient successfully.

Keywords Dumping syndrome · Intractable dumping syndrome · Laparoscopic Roux-en-Y gastric bypass · Modified duodenal switch

Conflict of Interest Chih-Kun Huang, Ming-Yu Wang, Ming-Che Hsin, and Po-Chih Chang have no conflict of interest.

Funding There is no external source of funding.

Statement of Informed Consent Informed consent was obtained from all individual participants included in the study.

Statement of Human and Animal Rights All procedures performed in studies involving human participants were in accordance with the ethical standards of the institutional and/or national research committee and with the 1964 Helsinki Declaration and its later amendments or comparable ethical standards. 